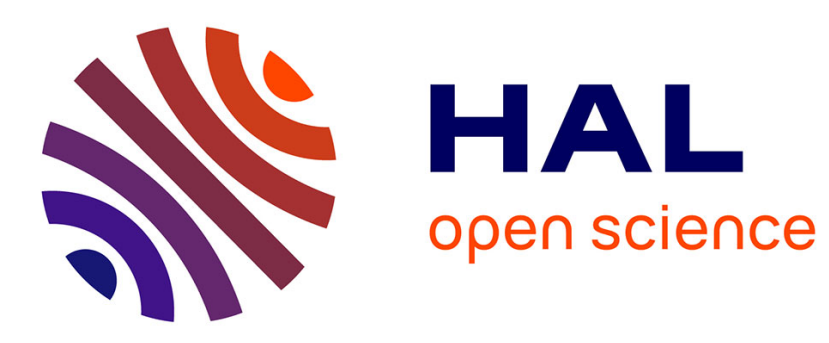

\title{
Model reduction techniques for quantitative nano-mechanical AFM mode
}

Xuyang Chang, Stéphane Roux, Simon Hallais, Kostas Danas

\section{To cite this version:}

Xuyang Chang, Stéphane Roux, Simon Hallais, Kostas Danas. Model reduction techniques for quantitative nano-mechanical AFM mode. Measurement Science and Technology, In press. hal-03174330

\author{
HAL Id: hal-03174330 \\ https://hal.science/hal-03174330
}

Submitted on 19 Mar 2021

HAL is a multi-disciplinary open access archive for the deposit and dissemination of scientific research documents, whether they are published or not. The documents may come from teaching and research institutions in France or abroad, or from public or private research centers.
L'archive ouverte pluridisciplinaire HAL, est destinée au dépôt et à la diffusion de documents scientifiques de niveau recherche, publiés ou non, émanant des établissements d'enseignement et de recherche français ou étrangers, des laboratoires publics ou privés. 


\title{
- Model reduction techniques for quantitative , nano-mechanical AFM mode
}

\author{
X. Chang ${ }^{1}$, S. Hallais ${ }^{1}$, S. Roux ${ }^{2}$ and K. Danas ${ }^{1}$ \\ ${ }^{1}$ LMS, C.N.R.S, École Polytechnique, Institut Polytechnique de Paris, \\ 91128 Palaiseau, France \\ ${ }^{2}$ Université Paris-Saclay/ENS Paris-Saclay/C.N.R.S., \\ LMT - Laboratoire de Mécanique et Technologie, 91190 Gif-sur-Yvette, France \\ E-mail: konstantinos.danas@polytechnique.edu \\ December 2020
}

\begin{abstract}
A recently developed atomic force microscope (AFM) process, the PeakForce Quantitative Nanomechanical Mapping (PF-QNM) mode, allows to probe over a large spatial region surface topography together with a variety of mechanical properties (e.g. apparent modulus, adhesion, viscosity). The resulting large set of data often exhibits strong coupling between material response and surface topography. This letter proposes the use of a proper orthogonal decomposition (POD) technique to analyze and segment the force-indentation data obtained by the PF-QNM mode in a highly efficient and robust manner. Two samples illustrate the proposed methodology. In the first one, low density polyethylene nanopods are deposited on a polystyrene film. The second is made of carbonyl iron particles embedded in a polydimethylsiloxane matrix. The proposed POD method permits to seamlessly identify the underlying phase constituents in both samples and decouple them from the surface topography by compressing voluminous force-indentation data into a subset with a much lower dimensionality.
\end{abstract}

Keywords : AFM; PeakForce-QNM; Segmentation; Model reduction technique; POD Submitted to: Meas. Sci. Technol. 


\section{Introduction}

Since Scanning Force Microscopy (SFM, aka SPM) was introduced [1], AFM has evolved into one of the most powerful tools for surface characterization [2]. Various new AFM modes has been proposed to provide local material properties together with topography with a high scanning rate (e.g., Tapping Mode [3], Pulse force Mode [4,5], Contact Resonance-AFM [6] and etc). Peak-Force Quantitative Nanomechanical Mapping (PFQNM) AFM mode has been introduced [7] as a new extension of previous Pulse Force AFM mode, aiming to robustly explore simultaneously various nanoscale mechanical properties [8-11]. By monitoring the instantaneous deflection of the cantilever, a continuous feedback loop is implemented to control the force between the tip and sample $[12,13]$. Force-indentation curves are generated separately for each tip oscillation (pixel by pixel) inside the region of interest (ROI), allowing to probe not only morphological properties (e.g. surface topography) but also various material mechanical properties such as Young's modulus, visco-elasticity, adhesion, or any indentation related properties [14-16].

In a PF-QNM analysis or any other type of micro/nano-indentation process, the measured force-indentation data involve the combined effect of sample topography, physical and chemical material properties [17-19], as well as the effective contact area between tip and sample. For most intrinsically hard materials (e.g. metals and ceramics), both the indentation size effect has been well investigated [20-24] and data analysis tools to estimate a reliable Young modulus has been estabilished [25, 26].

On the contrary, the lack of reliable nonlinear elastic contact models frequently compels the (inappropriate) use of Hertzian or Sneddon models to estimate the local apparent modulus and likely contributes to inconsistencies associated with the results of AFM measurements [27,28]. As a result, the mere use of the sole apparent modulus is insufficient to properly segment the phases in heterogeneous samples in a PF-QNM mode $[14,27]$. By contrast, use of the entire spatial and temporal force-indentation information may prove highly inefficient due to voluminous and overlapping data-sets that cannot be segmented properly and consequently lead to multiple fake material phases as a result of user-dependent segmentation processing.

From a data-mining perspective, the multi-dimensional character of the data does not allow for an intuitive and rigorous analysis [29], as compared to more classical twoand three-dimensional data spaces. In order to overcome the multi-dimensional and complex nature of the raw data obtained in a classical PF-QNM mode, it is proposed in this letter the use of a model reduction analysis such as the Proper Orthogonal Decomposition technique (POD [30] aka SVD [31] or PCA [32]). This technique allows to reorganize the data hierarchically, so that a mere truncation is a natural way to focus on the dominant features of the data-set, leaving aside higher-order information that contribute only weakly to the resulting force-indentation response at a given pixel. Furthermore, the truncation order is a choice that can be tuned if needed. This allows to clearly identify the underlying phases of the heterogeneous material and even decouple 
them from the surface topography, which usually interferes with the measured force response. In this view, the POD truncation is an effective method to convert the voluminous data-set into a subset with a much lower dimensionality and first-order information, where relevant features can be easily observed. Note however that the proposed approach is agnostic with respect to the physics of data. This makes the method highly versatile as no prior knowledge is encoded in the method, yet, it calls for a final physical, chemical and/or mechanical interpretation of the segmented data. This latter part is beyond the scope of the present letter and is left for a future study.

The efficiency of the proposed methodology is illustrated by two different heterogeneous samples. The first one consists of low density polyethylene (LDPE) canonical well-shaped disks, with no overlap, deposited on a polystyrene (PS) matrix, and is used as a patch test. The second sample comprises, in turn, hard micronsized carbonyl-iron particles (CIP) embedded into a polydimethylsiloxane (PDMS) matrix [33-36] leading to strong topography variations and non-trivial force-indentation spatial response.

\section{PeakForce QNM mode}

The experimental characteristics and output of the AFM PF-QNM mode are briefly described in Fig. 1. The laser spot is focused on the surface of the cantilever beam (Fig. 1a) and the associated probe measures the laser shifting voltage (LSV) over time, $\delta V(\boldsymbol{x}, t)$ at a given pixel on the surface described by the in-plane position vector $\boldsymbol{x}=(x, y)$. After a proper calibration process (usually performed on a nondeformable sapphire sample), the bending stiffness of the cantilever $\kappa$ and the sensitivity of the cantilever deflection $\gamma$ are estimated assuming a linear elastic, pure-bending response. This allows to directly associate the LSV measurement to the reaction force by $F(\boldsymbol{x}, t)=\kappa \delta V(\boldsymbol{x}, t)$ (Fig. 1b) and the cantilever deflection as $d_{\mathrm{df}}=\gamma \delta V(\boldsymbol{x}, t)$ (Fig. 1c). The actual indentation depth $\delta(\boldsymbol{x}, t)$ of the cantilever tip is given as the difference of the prescribed vertical displacement of the cantilever $Z$ (Fig. 1d) and the cantilever deflection as $d_{\mathrm{df}}, \delta(\boldsymbol{x}, t)=Z-d_{\mathrm{df}}$.

Use of $\delta$, instead of $Z$ or of time $t$, allows for the influence of topography to be erased for the most part. Fig. 1e shows a representative force-penetration, $F-\delta$, response at a fixed position (pixel) $\boldsymbol{x}$. The paths $\mathrm{A} \rightarrow \mathrm{B} \rightarrow \mathrm{C} \rightarrow \mathrm{D}$ (blue line) and $\mathrm{D} \rightarrow \mathrm{E} \rightarrow \mathrm{F}$ (red line) correspond to the loading and unloading response, respectively.

The entire $F-\delta$ response may then be divided in four main regimes (Fig. 1e):

- Regime I: $A \rightarrow B \rightarrow C$. As the tip approaches the surface of the specimen, an unstable jump towards contact occurs. The first force minimum during loading at $\mathrm{B}$ is used as a conventional definition of contact, and thus as an estimate of surface topography. However, because of the intrinsically unstable character of this "snapin" and its associated hysteresis [37], this commonly adopted definition appears to be delicate, and may intermingle topography with surface force gradients. An alternative more rigorous definition of topography may be obtained with regard to point $\mathrm{C}$, where 
(a)

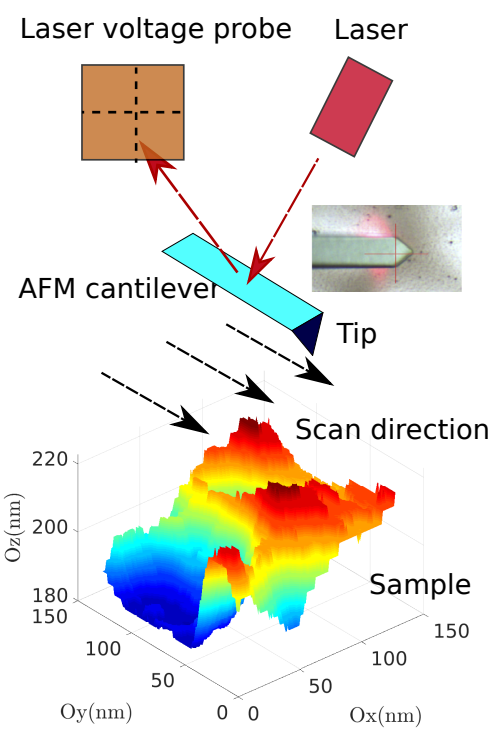

(b)
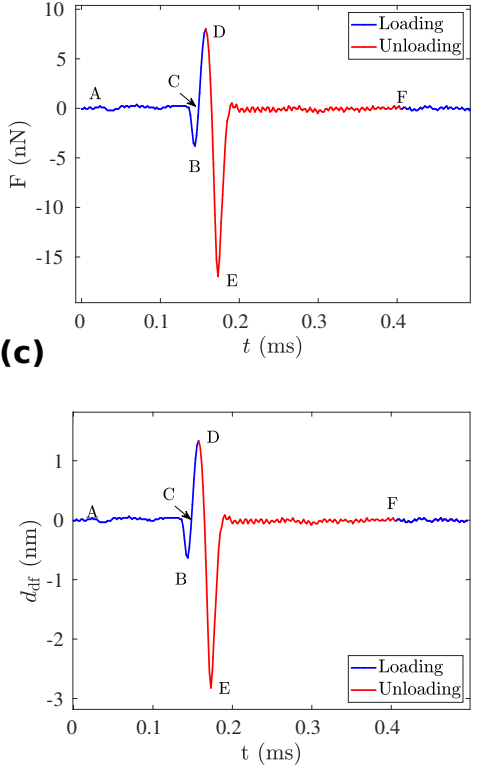

(d)

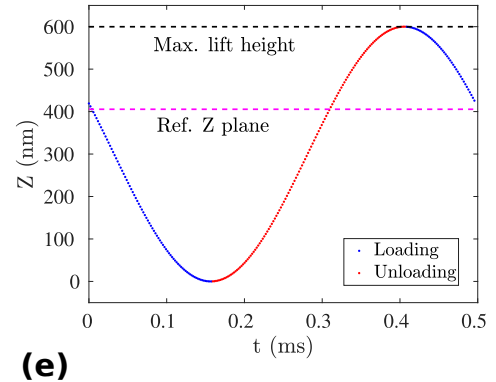

(e)

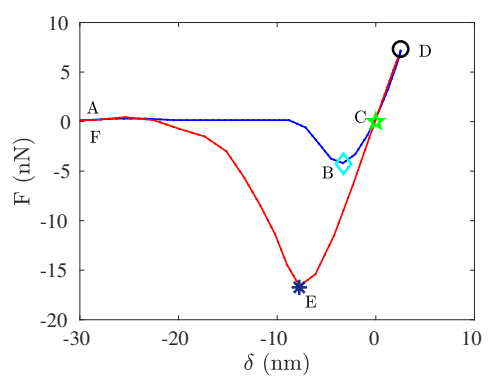

Figure 1: PeakForce QNM AFM mode. (a) AFM PeakForce QNM mode: a prescribed displacement loading is repeated in every pixel along the scanning direction; a laser beam reflected by AFM cantilever, is measured by a photo-diode delivering a laser shifting voltage (LSV), which can be converted in cantilever tip position and force at each instant in time $t$. (b) The deflection force $F$ vs. time $t$, (c) cantilever deflection $d_{\mathrm{df}}$ vs. time $t$, (d) total vertical displacement of the cantilever $Z$ vs. time $t$, (e) deflection force $F$ vs. actual indentation depth $\delta=Z-d_{\mathrm{df}}$. The markers denote the different regimes discussed in the main text.

the tip is in contact with the surface exerting a zero overall applied force. Thus the surface elevation $\delta_{e}(\mathbf{x})$ at a spatial point $\mathbf{x}$ is obtained by the implicit equation

$$
F\left(\mathbf{x}, \delta_{e}(\mathbf{x})=0\right)=0 \text {. }
$$

- Regime II: C $\rightarrow$ D. As the cantilever is pushed towards the surface, the force turns from attractive (before point $\mathrm{C}$ ) to repulsive (after point $\mathrm{C}$ ) and reaches a maximum at point D.

- Regime III: $\mathrm{D} \rightarrow \mathrm{E}$. The tip is then withdrawn (unloading), and the response is that of a (visco)-elastic adhesive contact. Adhesion can be characterized through the pull-out force $F$ reached at point $\mathrm{E}$ in the $F-\delta$ curve (dark blue star symbol).

- Regime IV: $\mathrm{E} \rightarrow \mathrm{F}$. Complete retraction of the tip is mainly dominated by the mechanical instability of tip detachment from the surface, similar to Regime I, but with a higher amplitude because of adhesion.

One often assumes that the sample remains purely elastic during the unloading cycle $\mathrm{D} \rightarrow \mathrm{E}$, so that an effective apparent Young's modulus can be estimated, using either a Hertz or a Sneddon contact model. Nonetheless, if nonlinear and/or viscous effects are present, this analysis can lead to erroneous results, as is the case here especially in the second PDMS-CIP sample. 


\section{Proper Orthogonal Decomposition}

This section discusses in some detail the proper orthogonal decomposition (POD) analysis used to analyze the force-indentation data obtained from the PF-QNM AFM mode. Initially introduced in Ref. [38] to study turbulence, the POD is a powerful and elegant method for data analysis aimed at obtaining low-dimensional approximate descriptions of a large data-set.

Specifically, in the present work, the force-indentation response is collected in a matrix form $\mathbf{F}\left(\mathbf{x}_{i}, \delta_{j}\right)$ written in index notation as $F_{i j}$. This matrix is sampled at each pixel position, $\mathbf{x}_{i},\left(i=1, . ., N_{x}\right.$ with $N_{x}$ denoting the number of pixels) and each indentation depth, $\delta_{j}\left(j=1, . ., N_{\delta}\right.$ with $N_{\delta}$ denoting the dimension of the indentation discretization). It should be noted here that linear interpolation between subsequent $\delta_{j}$ is required in general to obtain intermediate data necessary for the subsequent processes.

The POD analysis allows then to separate the matrix $\mathbf{F}$ into a set of orthonormal basis vectors (the POD modes) for representing a given data in the form

$$
\mathbf{F}(\mathbf{x}, \delta)=\sum_{n=1}^{N_{\delta}} \lambda^{(n)} \mathbf{U}^{(n)}(\mathbf{x}) \mathbf{W}^{(n)}(\delta), \quad \text { or } \quad F_{i j} \equiv \sum_{n=1}^{N_{\delta}} \lambda^{(n)} U_{i}^{(n)} W_{j}^{(n)} .
$$

Here, $\mathbf{W}^{(n)} \in \mathbb{R}^{N_{\delta}}$ represents the elementary force-indentation mode (normalized as $\left.\left\|\mathbf{W}^{(n)}\right\|=1 \ddagger\right), \mathbf{U}^{(n)} \in \mathbb{R}^{N_{x}}$ is the spatial modulation of this elementary response (normalized as $\left\|\mathbf{U}^{(n)}\right\|=1$ ) and $\lambda^{(n)}$ is a global modal amplitude. At this stage, no approximation is involved, and for all data series $\mathbf{F}(\mathbf{x}, \delta)$, such an exact space-indentation decomposition always exists (but is not unique).

Then, one may easily show that both the spatial and the force modes are orthogonal, i.e.,

$$
\mathbf{U}^{(n)} \cdot \mathbf{U}^{(m)}=\mathbf{W}^{(n)} \cdot \mathbf{W}^{(m)}=\delta^{(n m)}
$$

with $\delta^{(n m)}=1$ if $n=m$ and 0 otherwise. From the orthonormality conditions, the following relations can be readily derived

$$
\begin{aligned}
& \lambda^{(n)} \mathbf{U}^{(n)}=\mathbf{F} \mathbf{W}^{(n)} \\
& \lambda^{(n)} \mathbf{W}^{(n)}=\mathbf{U}^{(n)} \mathbf{F}
\end{aligned}
$$

Finally, the eigenvalues can be used to evaluate the relative "energy", $\tau_{n}$, of the $n$-th POD mode as

$$
\tau_{n}=\frac{\left(\lambda^{(n)}\right)^{2}}{\sum_{m}\left(\lambda^{(m)}\right)^{2}} .
$$

The most important property of the POD (that can be chosen as a definition) is the fact that modes can be ordered in terms of their significance for representing the $\ddagger$ We use a standard definition of the Euclidean vector norm $\|\mathbf{A}\|=\sqrt{\mathbf{A} \cdot \mathbf{A}}$, where $\mathbf{A}$ is a vector of any finite dimension. 
data. Then, one may retain only a very small number of those modes to approximate the original response. Both $\mathbf{U}^{(n)}$ and $\mathbf{W}^{(n)}$ as well as the number, $N \ll N_{\delta}$, of those retained modes are determined so that the norm of the difference between left and right hand side terms of Eq. (2) is minimized for any choice of $N$.

From the algorithmic point of view, $\lambda^{(n)}$ appear as eigenvalues sorted in descending order, whereas either $\mathbf{U}^{(n)}$ or $\mathbf{W}^{(n)}$ are the associated eigenvectors. Hence, the truncation of the above relation (Eq. 2) after the first $N<N_{\delta}$ modes,

$$
\widetilde{\mathbf{F}}_{N} \equiv \sum_{n=1}^{N} \lambda^{(n)} \mathbf{U}^{(n)}(\mathbf{x}) \mathbf{W}^{(n)}(\delta),
$$

provides the best approximation of the original data in a least squares sense for a given number of modes. As a result, the POD offers a simple way of compressing the data to a low dimensional space, while guaranteeing the optimality (or minimal loss) of such an approximation.

To estimate the accuracy of the approximate description obtained by the POD truncation, conventionally the residual $\rho_{i}$ at every spatial position $\mathbf{x}_{i}$ can be computed as

$$
\rho_{i}\left(\mathbf{x}_{i}\right) \equiv \rho_{i}=\frac{\sum_{j=1}^{N_{\delta}}\left(F_{i j}-\left(\widetilde{F}_{N}\right)_{i j}\right)^{2}}{\sum_{j=1}^{N_{\delta}}\left(F_{i j}\right)^{2}}, \quad i=1, . ., N_{x}
$$

\section{Patch-test: LDPE nano deposits on a PS film}

First, a sample made from low density polyethylene (LPDE) well-separated nanopods deposited on a polystyrene (PS) substrate is considered (Fig. 2)§. This sample serves as a patch test in our work since it is commonly used to calibrate AFM tips (RTESPA150 type). For the patch-test, a ROI area of $S=5 \times 5 \mu^{2}$ is scanned with a spatial resolution of $64 \times 64$ points and a frequency of acquisition $2 \mathrm{kHz}$.

Fig. $2 \mathrm{~b}$ shows the force-indentation response for twenty random selected pixels inside the ROI. It is clear that the corresponding force-indentation response can be divided into two main data-groups: the first one exhibits a stiff response with low adhesion and negligible viscosity, whereas the second one shows a softer response and with high adhesion and viscosity (as indicated by the hysteresis during unloading).

It is essential to point out that even if the PF-QNM mode is controlled to reach a predefined maximum contact force, this is in practice unattainable, as the scan frequency and the complex topography prevent this condition from being accurately satisfied. As a consequence, neither the force range nor the indentation interval are kept constant $\S$ The SEM image does not correspond exactly to the area analyzed by the AFM. Yet, it validates qualitatively the AFM results. 
(a)

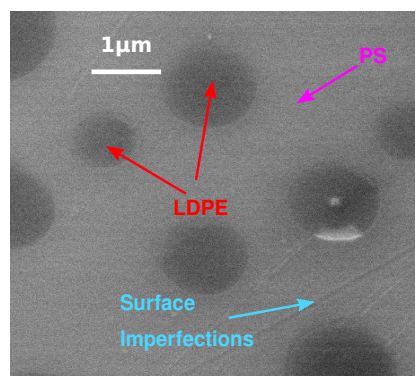

(d)

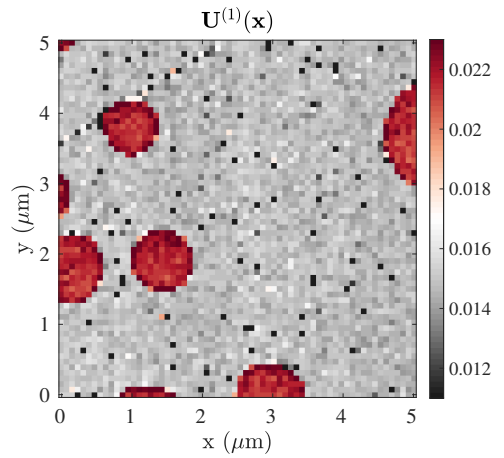

(g)

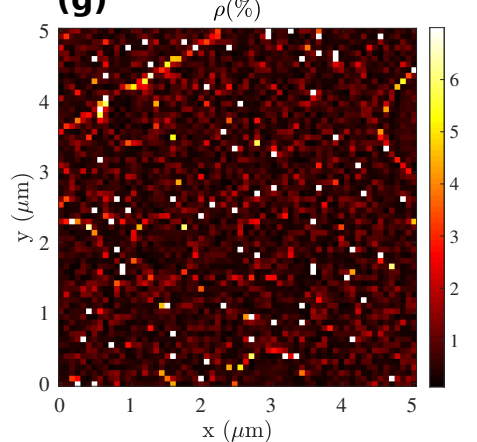

(b)

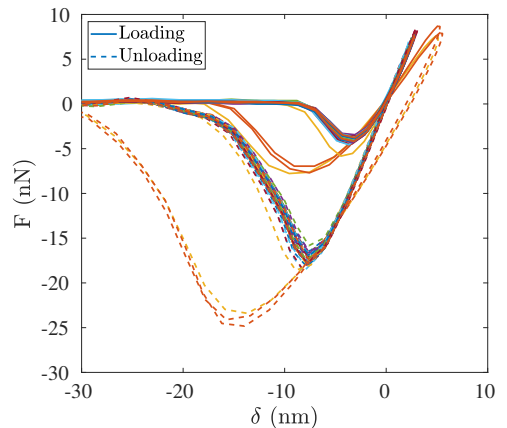

(e)

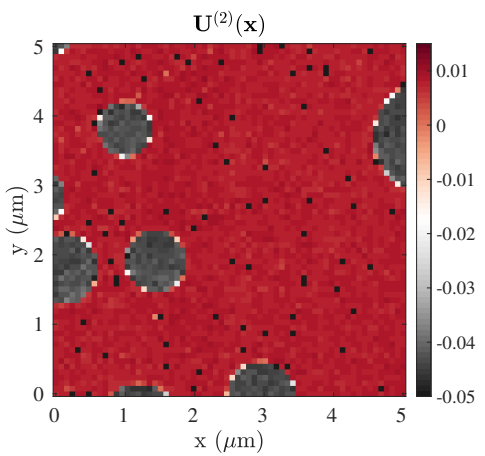

(h)

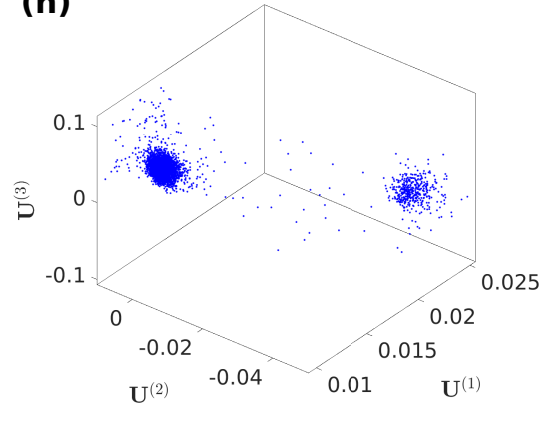

(c)

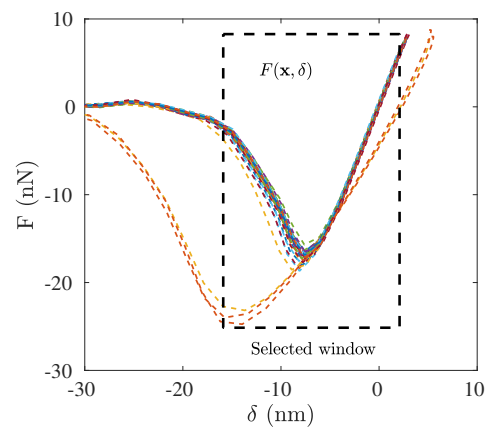

(f)

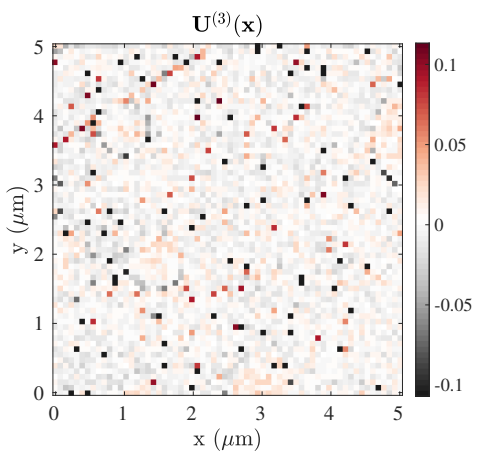

(i)

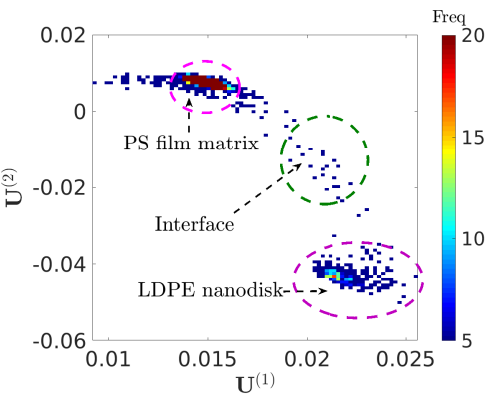

Figure 2: (a) SEM image showing LDPE nanopods deposited on PS substrate and surface imperfections. (b) Arbitrarily selected force-indentation response at various pixels (continuous lines represent tip approach and dashed lines tip retraction). (b) Force-indentation curve during retraction; the rectangle indicates the region selected for POD analysis. (d) First POD mode spatial mode revealing the phases (e) Second POD mode revealing more subtle information such as PS-LPDE interfaces. (c) Third POD mode showing higher order features related to surface roughness. (g) Residual error resulting by keeping only the first three modes to describe the force-indentation response at each pixel. (h) Subspace generated by the first three POD modes $\left[\mathbf{U}^{(1)} ; \mathbf{U}^{(2)} ; \mathbf{U}^{(3)}\right]$. (i) Contour of the frequency of points in the subspace $\left[\mathbf{U}^{(1)}, \mathbf{U}^{(2)}\right]$.

In this regard, for the patch test, the force-indentation response is analyzed only during unloading, i.e., Regime III, as shown by the cropping window in Fig. 2c (approximately $-15 \mathrm{~nm} \lesssim \delta \lesssim 5 \mathrm{~nm}$. The contact response is initially (visco)elastic 
and subsequently adhesive between the tip and the sample. This implies that our phase segmentation is done for this specific part of the $F-\delta$ response and has to be interpreted as such.

Subsequently, the cropped force-indentation data points are decomposed into $N$ POD (proper orthogonal decomposition) modes as described by Eq. (7). We show next that the first few POD modes can reproduce most of the complete $F-\delta$ response by evaluating the relative power of each POD mode $\tau_{n}$ in the original data is evaluated via Eq. (8).

Fig. 2(d-f) shows the first three POD spatial modes $\mathbf{U}^{(n)}\left(\mathbf{x}_{i}\right), n \leq 3$, ranked from higher to lower value of $\tau_{n}$. These first three POD modes represent $96 \%$ of the original measured $F-\delta$ response, leading respectively to the values, $\tau_{1}=0.75$, $\tau_{2}=0.17$, and $\tau_{3}=0.04$. The first POD mode $\mathbf{U}^{(1)}$ captures remarkably well the phase distributions (PS in gray and LDPE in light red in Fig. 2d) as the primary information of the mechanical response. The second mode, $\mathbf{U}^{(2)}$, (Fig. 2e) reveals the next level of information. In particular, light gray areas at the PS-LPDE interfaces indicates that the mechanical properties in those regions are somewhat different. Finally, the third mode $\mathbf{U}^{(3)}$ describes even higher order information that do not affect the first order effects such as the contact laws and material stiffness (Fig. 2f). For instance, the $\mathbf{U}^{(3)}$ map reveals regions with steep slopes, such as a scratch at the north-west side, which correlates well with similar defects revealed in the SEM image (Fig. 2a).

The contributions of higher POD modes, $n>N$, are negligible as compared to the first three ones and lead mostly to a pure noise map. In this view, the residual $\rho$ can be computed, to highlight pixels where the mechanical response is not very accurately accounted for with the number of POD modes used (Fig. 2g). For a more quantitative analysis, Fig. 2h shows the distribution of data in the subspace $\left[\mathbf{U}^{(1)} ; \mathbf{U}^{(2)} ; \mathbf{U}^{(3)}\right]$, where pixels are grouped into clusters. This allows the segmentation of the different phases and the identification of one or more interfacial regions. Focusing further in the subspace $\left[\mathbf{U}^{(1)} ; \mathbf{U}^{(2)}\right]$ (Fig. 2i), a 2D-histogram shows the statistical frequency of points having a given value of $\mathbf{U}^{(1)}$ and $\mathbf{U}^{(2)}$. Two main phases characterized by their mean response and deviations are very clearly highlighted making mechanically-based segmentation quite simple.

\section{Carbon-Iron particles with PDMS binder}

The second analyzed sample is a composite material consisting of a polymer matrix (PDMS) and mechanically stiff, fairly spherical carbonyl-iron particles (CIP) with mean radius of about $\sim 3 \mu \mathrm{m}$. The results from the built-in QNM results are first shown in Fig. 3 to reveal the complexity of the analyzed sample. Subsequently, in Fig. 4, we analyze the date using the proposed POD method.

As seen in Fig. 3a obtained by SEM, the white spots represent the reflections from the CIP, whereas the surface of the composite material is marked by multiple line defects. For the AFM analysis, a surface of $50 \mathrm{\mu m}^{2}$ is scanned using the PF-QNM mode with a 
(a)

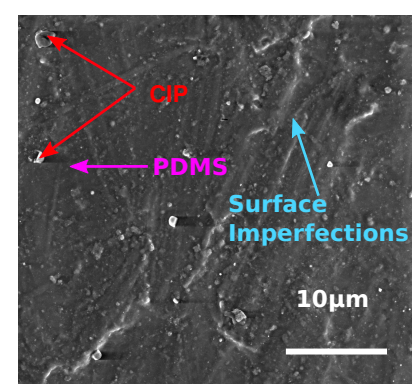

(b)

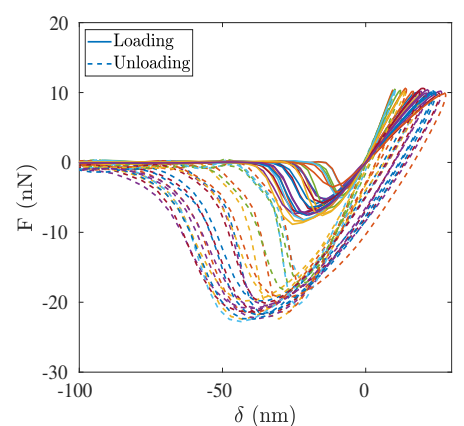

(d)

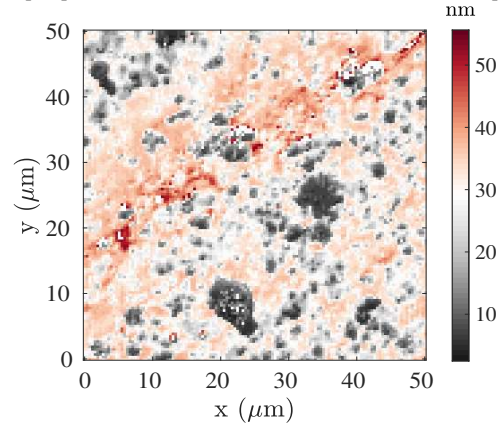

(e)

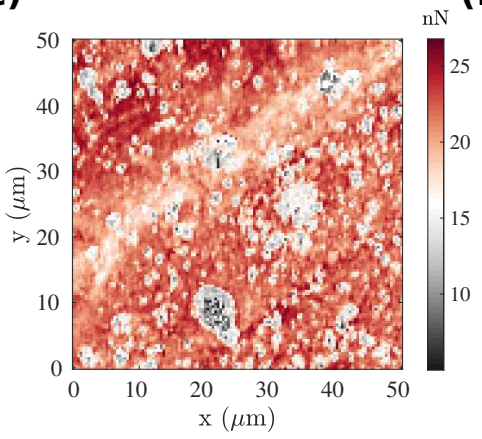

(c)

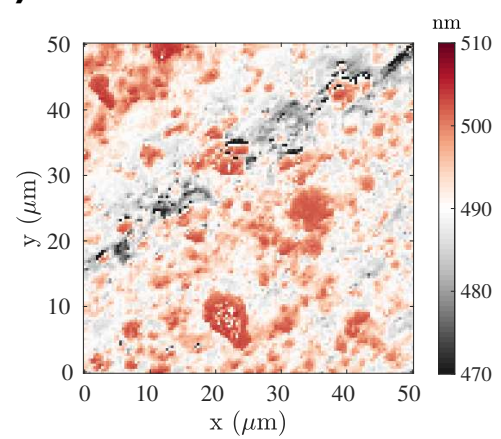

(f)

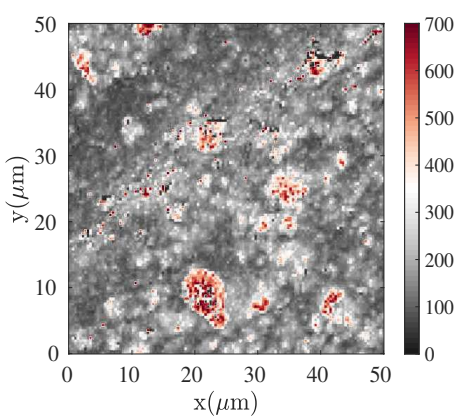

Figure 3: (a) SEM image showing carbonyl iron particles (CIP) embedded in a PDMS matrix, and surface imperfections. (b) Arbitrarily selected force-indentation response at various pixels (continuous lines represent tip approach and dashed lines tip retraction). (c) Topography map using our proposed definition; (d)-(f) Bruker's PF-QNM built-in results maximum indentation; (d) Maximum Indentation (e) Adhesion (f) Apparent modulus using Sneddon model;

Following the same POD procedure presented in the previous section, a window is selected in the unloading Regime III (Fig. 4a) with $\delta$ ranging from $\sim-45 \mathrm{~nm}$ to $\sim 10 \mathrm{~nm}$. In this initial data-set, after the POD analysis, the first three modes are retained, as shown in Fig. 4(b-d). Their contribution amounts to $\tau_{1}=0.91>\tau_{2}=0.04>\tau_{3}=0.03$, respectively, describing approximately $98 \%$ of the power of the original $F-\delta$ data. 
(a)

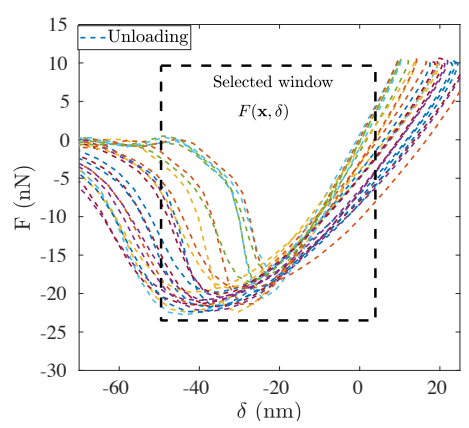

(d)

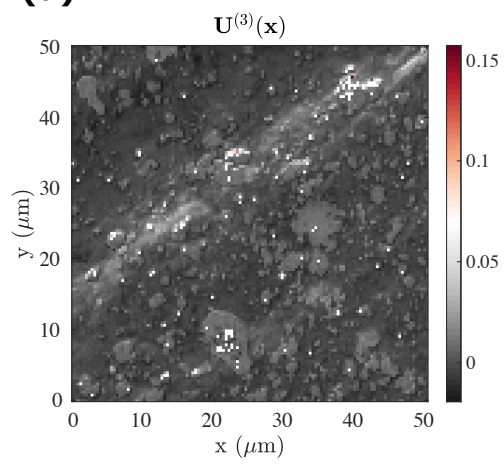

(b)

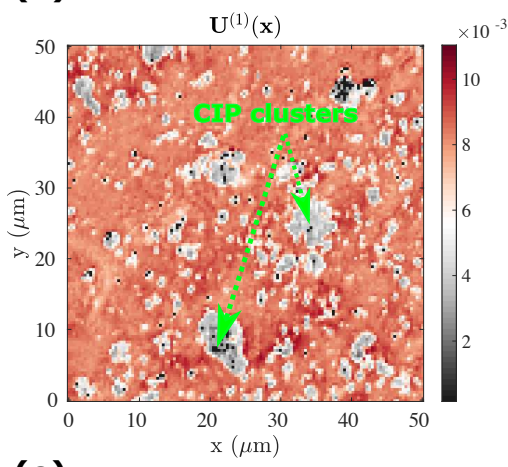

(e)

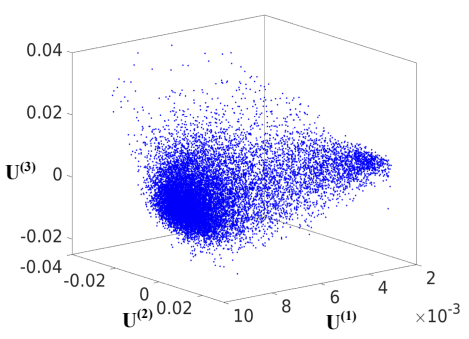

(c)

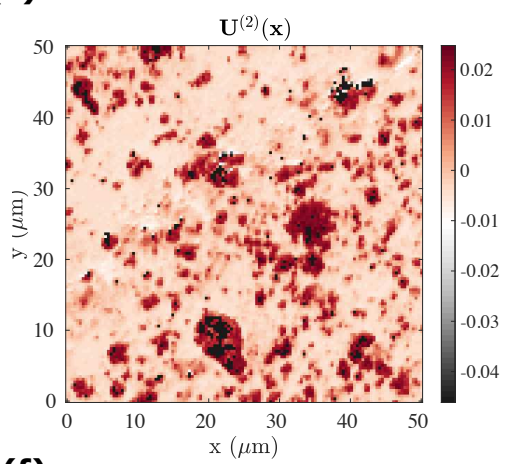

(f)

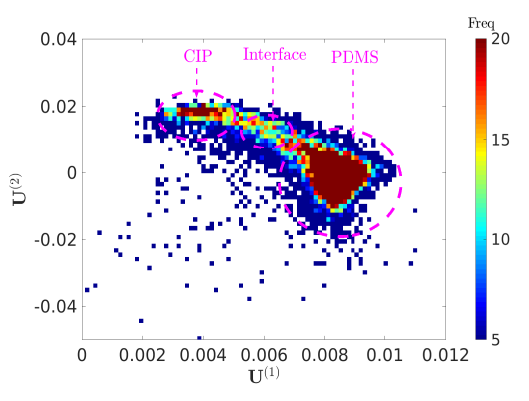

Figure 4: (a) Force-indentation curve during retraction; the rectangle indicates the region selected for POD analysis. (b)-(c) First two POD mode spatial modes revealing clearly the CIP-PDMS phases (d) Third POD mode showing higher order features related to surface roughness.(e) Subspace generated by the first three POD modes $\left[\mathbf{U}^{(1)} ; \mathbf{U}^{(2)} ; \mathbf{U}^{(3)}\right]$. (f) $2 \mathrm{D}$ histogram of amplitudes in the subspace $\left[\mathbf{U}^{(1)}, \mathbf{U}^{(2)}\right]$.

Remarkably, despite the continuous pattern in the $F-\delta$ responses (Fig. 4a), the first mode $\mathbf{U}^{(1)}$ (Fig. 4b) reveals the presence of the PDMS matrix (in red) contrasting with the much smaller amplitude of the stiff CIP phase (in white). In particular, we observe a pronounced clustering of CIP particles in at least four regions that exceed a side length of $10 \mu \mathrm{m}$ (i.e., 3-4 times the radius if the particle) due to aggregation during the sample fabrication. Fig. 4b illustrates the strength of the AFM-POD analysis as compared with the SEM imaging, wherein such delicate features are much more difficult to obtain.

The second mode (Fig. 4c) in the present case does not exhibit substantially different features than the first one. In fact, one may note that the CIP particles now have a much larger weight than the soft matrix, i.e.opposite to the case of the first mode. With our proposed algorithm, the rough surface topography does not appear to bias the phase contrast seen in Fig. $4 \mathrm{~b}$ and c. By contrast, the surface topography is mingled with the phase contrast in all the different Bruker outputs in Fig. 3. Thus, it may be concluded that in the present examples, the POD analysis is a trustworthy and efficient method for phase segmentation.

Finally, the third mode (Fig. 4d) reveals the next order of information, this time highlighting the aforementioned topographical defect (light white color) ranging from 
south-west to north-east. In the literature, the influence of topography on apparent adhesion has been intensively studied. A sharp variation in surface curvature often leads to a decrease in adhesion for the same material [39]. This observation is consistent with the results reported here as well as those processed by the Bruker AFM software, in spite of the fact that the first modes were observed to be independent of topography. Hence, the POD analysis appears to be an efficient method for rearranging hierarchically and separately different features in PF-QNM AFM data (phase, topography) according to their contribution in the mechanical signal, allowing analysts to describe each individual aspects or their combination altogether.

Focusing, next, on the reduced subspace $\left[\mathbf{U}^{(1)} ; \mathbf{U}^{(2)} ; \mathbf{U}^{(3)}\right]$ allows to reveal the continuous distribution of the data (Fig. 4e). Given that topography is almost entirely suppressed in the first two POD modes, the subspace $\left[\mathbf{U}^{(1)} ; \mathbf{U}^{(2)}\right]$, (Fig. 4f), becomes a natural "best-candidate" for the purpose of phase segmentation. Two distinct peaks, corresponding to the two main phases, i.e, PDMS and CIP can be observed. However, the scatter of points and the overlap of the two domains suggests in this case that the transition (in terms of apparent mechanical properties) is progressive. It may be speculated that particles buried at different depth beneath the surface may be responsible for this observation.
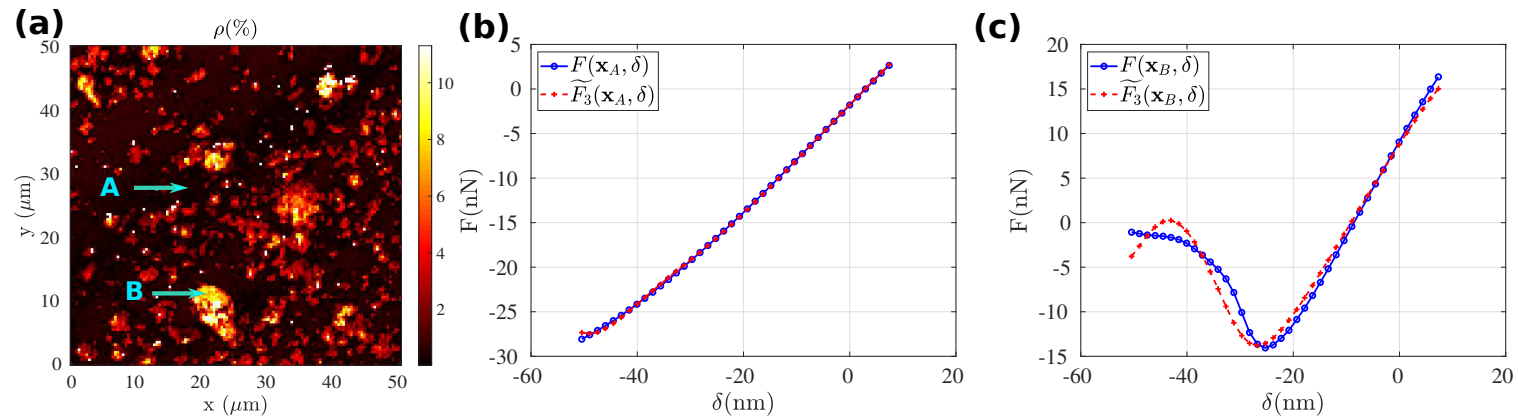

Figure 5: Uncertainty(residual) of reconstruction using the first three POD modes: (a) The residual map ; (b) Comparison between the initial force-indentation curve (plotted in dot blue) and reconstructed curve (plotted in red) at point $\mathrm{A}\left(\rho\left(\mathbf{x}_{A}\right) \simeq 1 \%\right)$; (c) Comparison between the initial force-indentation curve (plotted in dot blue) and reconstructed curve (plotted in red) at point $\mathrm{B}\left(\rho\left(\mathbf{x}_{B}\right) \simeq 10 \%\right)$

Finally, in order to assess the accuracy of the POD reconstruction, we show in Fig. 5a, the residual, $\rho$, which serves to measure the error induced by the truncation to only the first three modes at each pixel. This measure suggests that CIP clusters may require a finer analysis to be better described. In particular, we select and analyze two points with different residual levels, as shown in Fig. 5a. At point A located in the PDMS matrix (see Fig. 5b), the initial force curve is perfectly reconstructed with an error that is less than $1 \%$. In contrast, at point B located inside a cluster of CIP particles, (Fig. 5c), the truncation error (of the order of $\simeq 10 \%$ ) is mostly concentrated at the maximum pull-out force. One possible explanation is that the error results from the unstable mechanism of 'snap-off' between tip and sample. However, the hysteretic 
mechanism of 'snap-off' is out of the scope of this study, and thus we did not further attempt to reduce the reconstruction error by introducing additional higher order POD modes.

\section{Conclusions}

Accessing complex nano- and microstructural morphologies in heterogeneous media is both a need and a challenge. The recent PF-QNM AFM mode represents a major step forward to provide such fine information, whereby each image pixel is fully characterized by a complete mechanical test. However, the analysis of the resulting large data-sets becomes not only delicate (because of the intrinsic coupling of different mechanical and chemical properties with the topography), but also time-wise prohibitive. This letter has shown that model reduction techniques (such as the POD), can be extremely useful in organizing hierarchically such large data-sets allowing not only to identify a small number of modes expressing the underlying phases but also to offer an easy segmentation of the (mechanically relevant) phases. Starting from the force-indentation response, proper classification may reveal discrete material responses, allowing to extract seamlessly the mechanical, chemical or physical response of each of them. In materials with complex microstructures, the proposed processing may indicate, at first sight, that mechanical properties are continuously varying making a manual identification impossible. The POD method allows to properly identify the data points belonging to the same phase and possibly to a transition region between them.

We close by emphasizing that the agnostic character of the data processing techniques used here is both a strength - no bias is introduced by enforcing say a contact model that would be unsuited - and a weakness - the physical interpretation (e.g. elastic stiffness, adhesive properties, viscoelasticity) remains in the hand of the user. However, this interpretation becomes now substantially easier and more robust since only a reduced subspace of a much lower dimensionality (i.e.modes) needs to be considered.

\section{Acknowledgement}

X.C., S.H. and K.D. would like to thank Becton and Dickinson Corporation (BD) for financing partially this project and Ms. Saphia Ouanani from Bruker Corporation for very useful discussions. K.D. would also like to acknowledge partial support from the European Research Council (ERC) under the European Union's Horizon 2020 research and innovation program (grant agreement No 636903). 
Model reduction techniques for quantitative nano-mechanical AFM modes

\section{Appendix A.2. QNM properties}

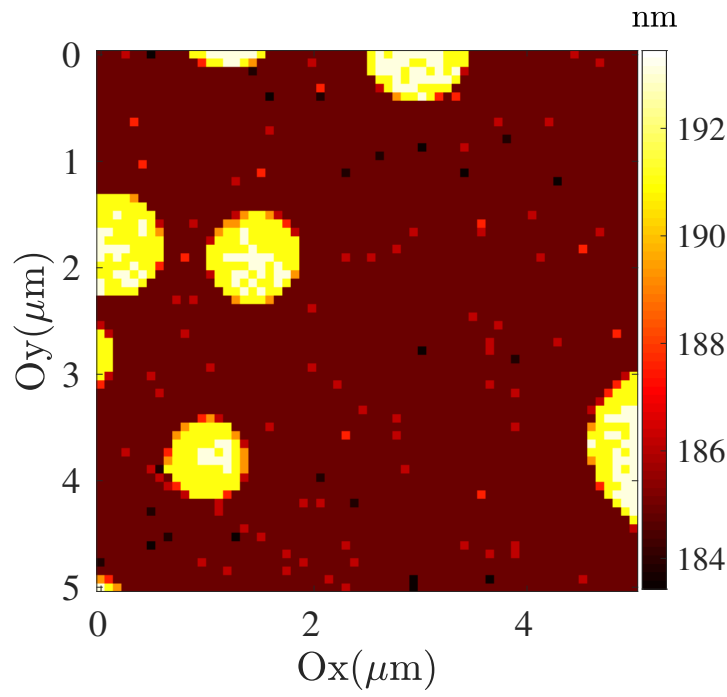

(a)

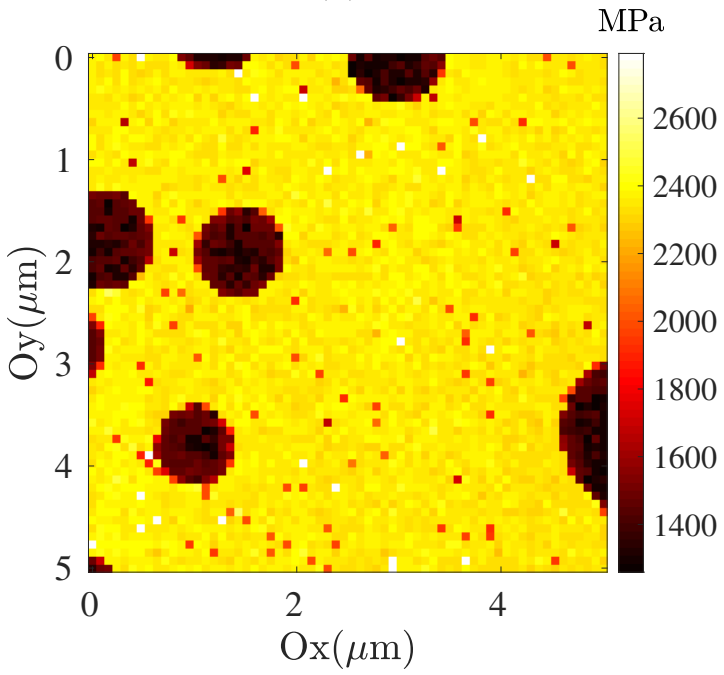

(c)

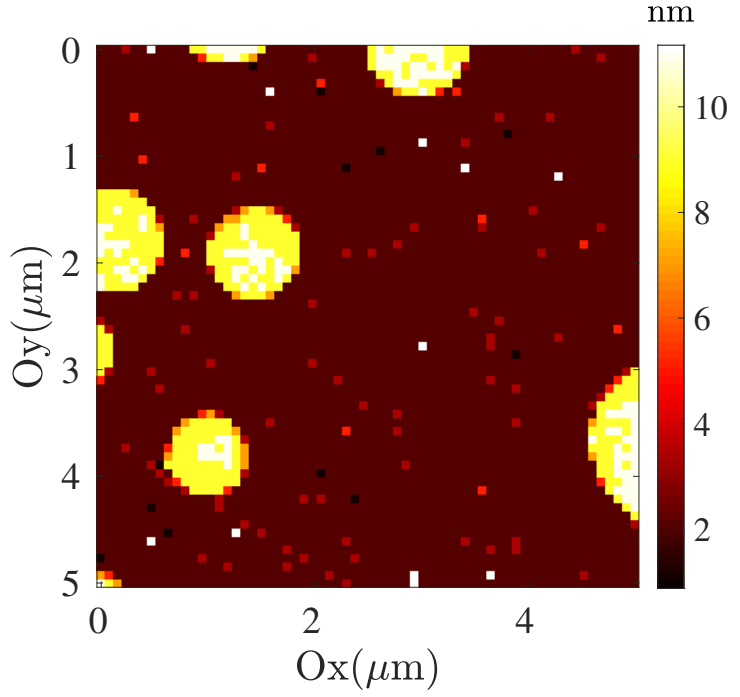

(b)

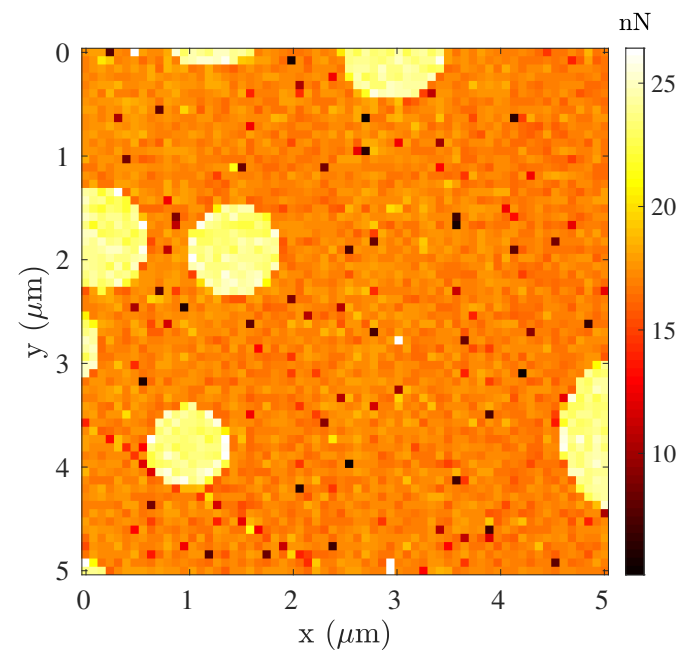

(d)

Figure A1: The PF-QNM modality proposed by Bruker provides different mechanical characterizations based on the AFM scan discussed in the main text of the manuscript, relative to the PS-LDPE sample. (a) Topography map; (b) Maximum indentation; (c) Apparent modulus using Hertz model; (d) Adhesion 


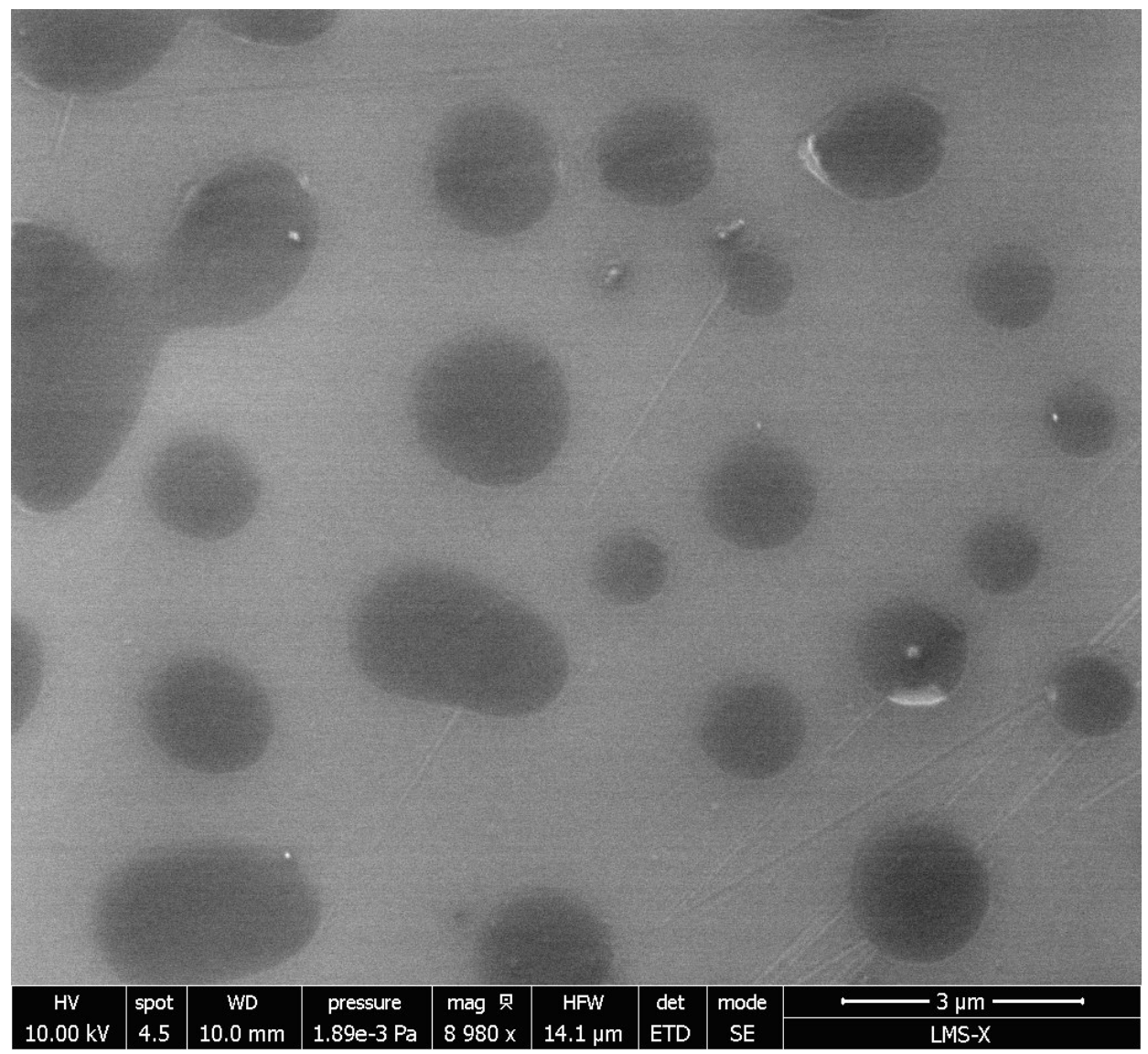

Figure A2: SEM image (secondary electrons) for the PS-LDPE sample (the dark gray domains are the LDPE nanopods while the PS film substrate appears in light gray 


\section{Appendix B. PDMS-CIP sample}

\section{Appendix B.1. Fabrication process}

The fabrication procedure of the PDMS + CIP composite can be summarized as follows (see more details in [34]):

1. The appropriate amount of CIP powder is mixed along with part A + part B (10:1) of Sylgard 184 in a beaker.

2. All ingredients are thoroughly mixed for two minutes at 200 RPM mixer.

3. The mixture is put into a vacuum chamber for 34 minutes to remove the entrapped air.

4. The degassed liquid mixture is put in an aluminum mold.

5. The mold is heated in an oven at temperature $T=373 \mathrm{~K}$ for two hours.

Appendix B.2. SEM image

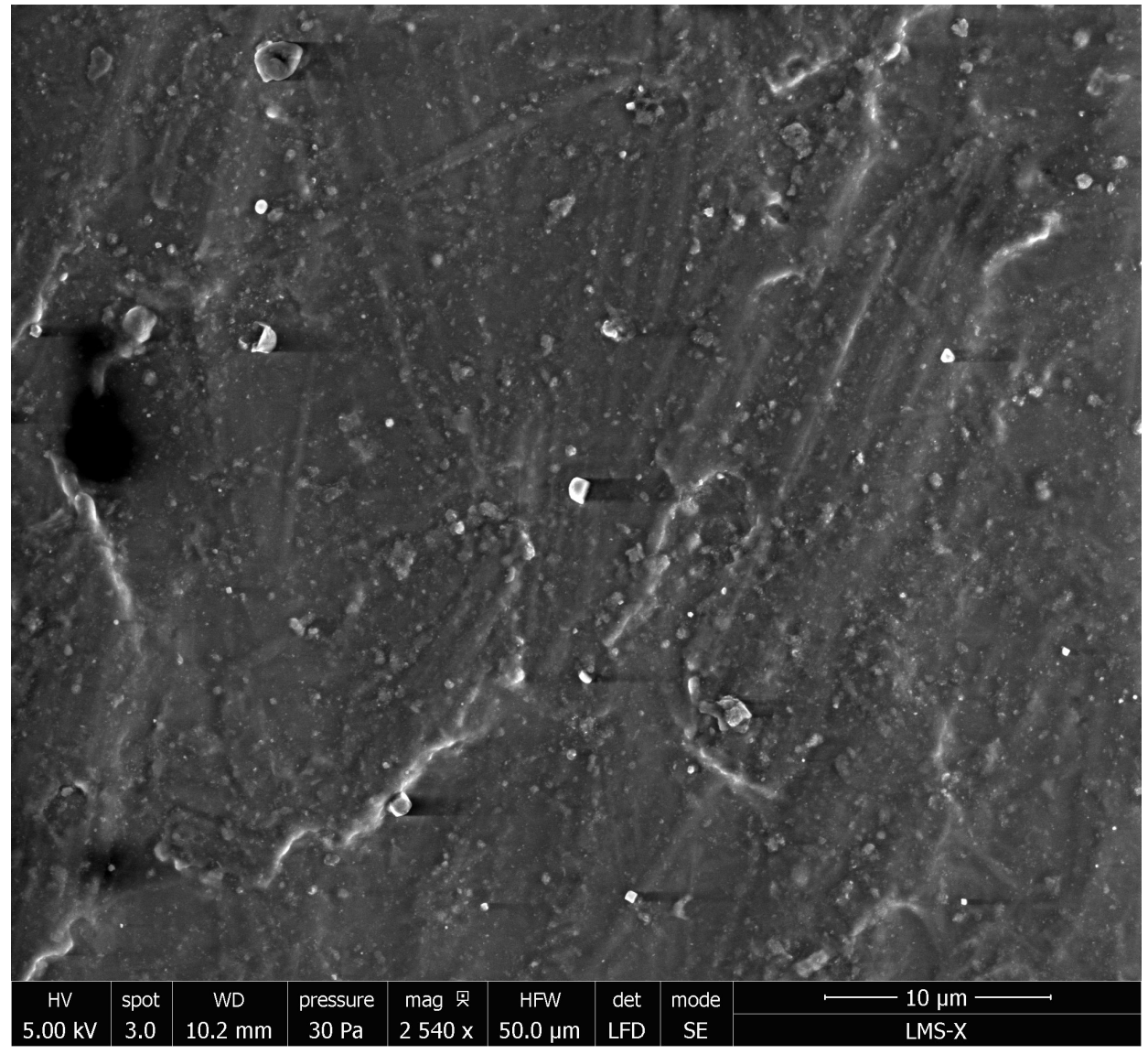

Figure B1: SEM image (secondary electrons) for the PDMS-CIP sample. Bright spots originates from the Carbonyl-Iron particles, while the PDMS shows a darker gray level. A significant roughness of the surface is visible 


\section{Appendix C. POD truncation}

Note that $\mathbf{F}$ by construction is not a square (and hence not symmetric). In order to accelerate the computations, we symmetrize $\mathbf{F}$ in order to form a square matrix of a minimum dimension that allows to obtain seamlessly the eigenvalues $\lambda^{(n)}$ and the eigenvectors $\mathbf{W}^{(n)}$. In the present work, we always have $N_{\delta}<N_{x}$. As a consequence, the most efficient symmetrization is obtained by setting [30],

$$
\mathbf{M}=\mathbf{F}^{T} \mathbf{F}, \quad \text { or } \quad M_{i j}=\sum_{k=1}^{N_{x}} F_{k j} F_{k i} .
$$

This operation leads to a matrix $\mathbf{M}$ of size $N_{\delta} \times N_{\delta}$. The alternative one $\mathbf{F} \cdot \mathbf{F}^{T}$ would lead to a matrix size of dimension $N_{x} \times N_{x}>N_{\delta} \times N_{\delta}$. Using now the definition introduced in Eq. (2) and simple linear algebra, we can readily get

$$
M_{i j}=\sum_{n=1}^{N_{\delta}}\left(\lambda^{(n)}\right)^{2} W_{i}^{(n)} W_{j}^{(n)} .
$$

Thus, use of the symmetric (square) matrix $\mathbf{M}$ instead of the non-symmetric $\mathbf{F}$ allows to extract in a very simple manner the eigenvalues $\lambda^{(n)}$ and eigenvectors $\mathbf{W}^{(n)}$ by employing any eigensystem algorithm for symmetric real matrices. Once those two quantities are evaluated, one may extract the remaining spatial modes $\mathbf{U}^{(n)}$ by use of the orthogonality between the $\mathbf{W}^{(n)}$ modes and the direct projection operation, described in Eq. (4).

The following algorithm describes the POD operations using this last definition as well as the definitions in Section 3.

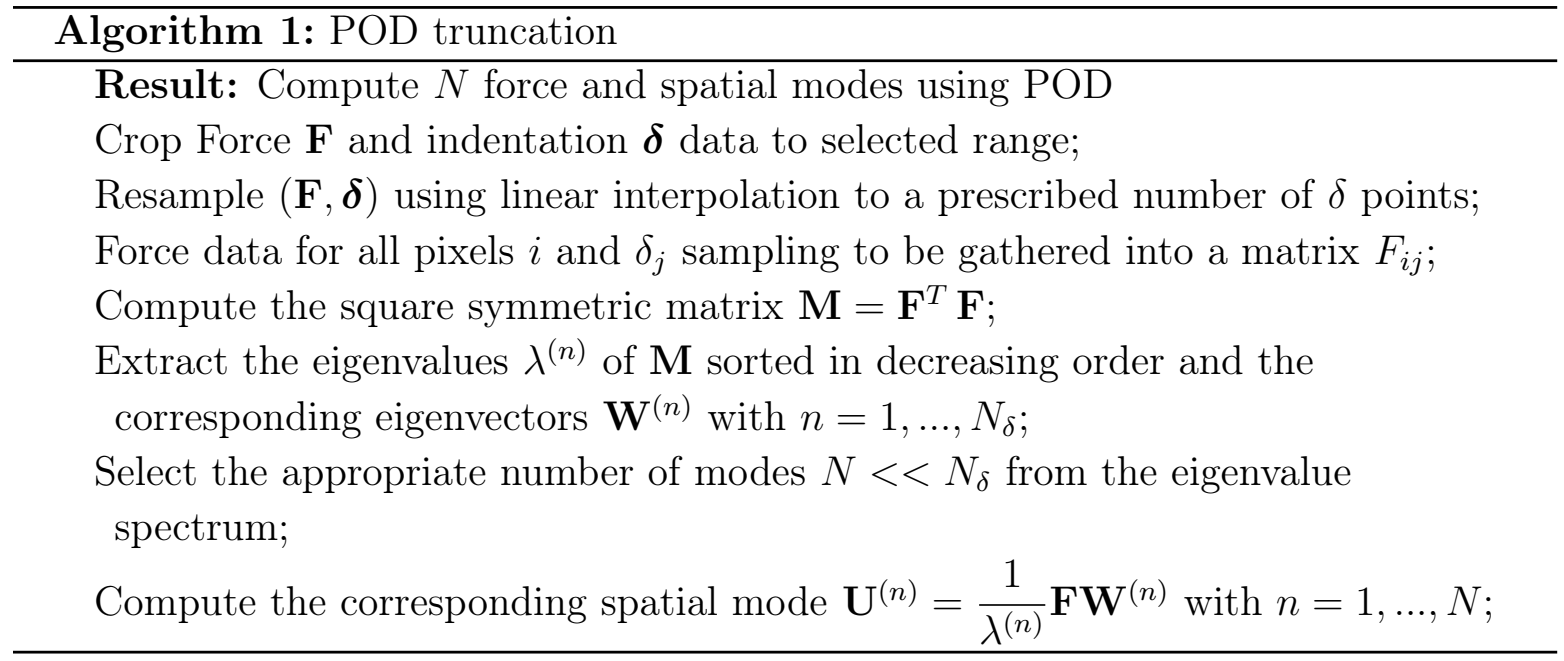


[1] G. Binnig, C. F. Quate, and Ch. Gerber. Atomic force microscope. Phys. Rev. Lett., 56:930-933, Mar 1986.

[2] C.F Quate. The afm as a tool for surface imaging. Surface Science, 299-300:980 - 995, 1994.

[3] S.N. Magonov, V. Elings, and M.-H. Whangbo. Phase imaging and stiffness in tapping-mode atomic force microscopy. Surface Science, 375(2):L385 - L391, 1997.

[4] A Rosa-Zeiser, E Weilandt, S Hild, and O Marti. The simultaneous measurement of elastic, electrostatic and adhesive properties by scanning force microscopy: pulsed-force mode operation. Measurement Science and Technology, 8(11):1333-1338, nov 1997.

[5] Lining Lan, Shuhong Xie, Li Tan, and Jiangyu Li. Sol-gel based soft lithography and piezoresponse force microscopy of patterned $\mathrm{pb}(\mathrm{zrti})$ microstructures. Journal of Materials Science $\mathcal{G}^{3}$ Technology, 26(5):439 - 444, 2010.

[6] D. Passeri, M. Rossi, and J.J. Vlassak. On the tip calibration for accurate modulus measurement by contact resonance atomic force microscopy. Ultramicroscopy, 128:32 - 41, 2013.

[7] T J Young, M A Monclus, T L Burnett, W R Broughton, S L Ogin, and P A Smith. The use of the PeakForceTMquantitative nanomechanical mapping AFM-based method for highresolution young's modulus measurement of polymers. Measurement Science and Technology, 22(12):125703, oct 2011.

[8] Kim K. M. Sweers, Kees O. van der Werf, Martin L. Bennink, and Vinod Subramaniam. Atomic force microscopy under controlled conditions reveals structure of c-terminal region of $\alpha$-synuclein in amyloid fibrils. ACS Nano, 6(7):5952-5960, 2012. PMID: 22695112.

[9] Maxim E. Dokukin and Igor Sokolov. Quantitative mapping of the elastic modulus of soft materials with harmonix and peakforce qnm afm modes. Langmuir, 28(46):16060-16071, 2012. PMID: 23113608

[10] Moritz Pfreundschuh, David Alsteens, Manuel Hilbert, Michel O. Steinmetz, and Daniel J. Müller. Localizing chemical groups while imaging single native proteins by high-resolution atomic force microscopy. Nano Letters, 14(5):2957-2964, 2014. PMID: 24766578.

[11] Hsien-Shun Liao, Ka Kit Lei, and Yu Fang Tseng. High-speed force mapping based on an astigmatic atomic force microscope. Measurement Science and Technology, 30(2):027002, jan 2019.

[12] Franz J. Giessibl. Advances in atomic force microscopy. Rev. Mod. Phys., 75:949-983, Jul 2003.

[13] Yueming Hua. PeakForce-QNM advanced applications training 2014. Technical Support Engineer, 2012.

[14] G. Smolyakov, S. Pruvost, L. Cardoso, B. Alonso, E. Belamie, and J. Duchet-Rumeau. Afm peakforce qnm mode: Evidencing nanometre-scale mechanical properties of chitin-silica hybrid nanocomposites. Carbohydrate Polymers, 151:373 - 380, 2016.

[15] Laida Cano, Daniel Humberto Builes, Sheyla Carrasco-Hernandez, Junkal Gutierrez, and Agnieszka Tercjak. Quantitative nanomechanical property mapping of epoxy thermosetting system modified with poly(ethylene oxide-b-propylene oxide-b-ethylene oxide) triblock copolymer. Polymer Testing, 57:38 - 41, 2017.

[16] M. Majewska, D. Mrdenovic, I.S. Pieta, R. Nowakowski, and P. Pieta. Nanomechanical characterization of single phospholipid bilayer in ripple phase with pf-qnm afm. Biochimica et Biophysica Acta (BBA) - Biomembranes, 1862(9):183347, 2020.

[17] E. Barthel and S. Roux. Velocity-dependent adherence: An analytical approach for the jkr and dmt models. Langmuir, 16(21):8134-8138, 2000.

[18] Gregory D. Jay, Jahn R. Torres, David K. Rhee, Heikki J. Helminen, Mika M. Hytinnen, ChungJa Cha, Khaled Elsaid, Kyung-Suk Kim, Yajun Cui, and Matthew L. Warman. Association between friction and wear in diarthrodial joints lacking lubricin. Arthritis 8 Rheumatism, 56(11):3662-3669, 2007.

[19] Qunyang Li and Kyung-Suk Kim. Micromechanics of friction: effects of nanometre-scale roughness. Proceedings of the Royal Society A: Mathematical, Physical and Engineering Sciences, 464(2093):1319-1343, 2008. 
[20] Uzi Landman, W. D. Luedtke, Nancy A. Burnham, and Richard J. Colton. Atomistic mechanisms and dynamics of adhesion, nanoindentation, and fracture. Science, 248(4954):454-461, 1990.

[21] N.A. Fleck, G.M. Muller, M.F. Ashby, and J.W. Hutchinson. Strain gradient plasticity: Theory and experiment. Acta Metallurgica et Materialia, 42(2):475 - 487, 1994.

[22] William D. Nix and Huajian Gao. Indentation size effects in crystalline materials: A law for strain gradient plasticity. Journal of the Mechanics and Physics of Solids, 46(3):411 - 425, 1998.

[23] G. Haiat, M.C. Phan Huy, and E. Barthel. The adhesive contact of viscoelastic spheres. Journal of the Mechanics and Physics of Solids, 51(1):69 - 99, 2003.

[24] E Barthel. Adhesive elastic contacts: Jkr and more. Journal of Physics D: Applied Physics, 41:163001, 2008.

[25] David Lin, Emilios Dimitriadis, and Ferenc Horkay. Robust strategies for automated afm force curve analysis - i. non-adhesive indentation of soft, inhomogeneous materials. Journal of biomechanical engineering, 129:430-40, 072007.

[26] David Lin, Emilios Dimitriadis, and Ferenc Horkay. Robust strategies for automated afm force curve analysis - ii: Adhesion-influenced indentation of soft, elastic materials. Journal of biomechanical engineering, 129:904-12, 012008.

[27] David C. Lin, David I. Shreiber, Emilios K. Dimitriadis, and Ferenc Horkay. Spherical indentation of soft matter beyond the hertzian regime: numerical and experimental validation of hyperelastic models. Biomechanics and Modeling in Mechanobiology, 8(5):345, Nov 2008.

[28] B J Briscoe, L Fiori, and E Pelillo. Nano-indentation of polymeric surfaces. Journal of Physics D: Applied Physics, 31(19):2395-2405, oct 1998.

[29] Michel Verleysen and Damien François. The curse of dimensionality in data mining and time series prediction. volume 3512, pages 758-770, 062005.

[30] Anindya Chatterjee. An introduction to the proper orthogonal decomposition. Current science, pages $808-817,2000$.

[31] Charles F Van Loan. Generalizing the singular value decomposition. SIAM Journal on numerical Analysis, 13(1):76-83, 1976.

[32] Svante Wold, Kim Esbensen, and Paul Geladi. Principal component analysis. Chemometrics and intelligent laboratory systems, 2(1-3):37-52, 1987.

[33] K. Danas, S.V. Kankanala, and N. Triantafyllidis. Experiments and modeling of iron-particlefilled magnetorheological elastomers. Journal of the Mechanics and Physics of Solids, 60(1):120 $-138,2012$.

[34] L. Bodelot, J.-P. Voropaieff, and T. Pössinger. Experimental investigation of the coupled magnetomechanical response in magnetorheological elastomers. Experimental Mechanics, 58(2):207-221, sep 2017.

[35] Erato Psarra, Laurence Bodelot, and Kostas Danas. Two-field surface pattern control via marginally stable magnetorheological elastomers. Soft Matter, 13(37):6576-6584, 2017.

[36] E. Psarra, L. Bodelot, and K. Danas. Wrinkling to crinkling transitions and curvature localization in a magnetoelastic film bonded to a non-magnetic substrate. Journal of the Mechanics and Physics of Solids, 133:103734, 2019.

[37] Sebastian Rützel, Soo Il Lee, and Arvind Raman. Nonlinear dynamics of atomic force microscope probes driven in lennard jones potentials. Proceedings of the Royal Society of London. Series A: Mathematical, Physical and Engineering Sciences, 459(2036):1925-1948, 2003.

[38] K. K. Nomura and S. E. Elghobashi. The structure of inhomogeneous turbulence in variable density nonpremixed flames. Theoretical and Computational Fluid Dynamics, 5(4):153-175, Nov 1993.

[39] T. Stifter, E. Weilandt, O. Marti, and S. Hild. Influence of the topography on adhesion measured by sfm. Applied Physics A, 66(1):S597-S605, Mar 1998. 